\title{
THE PADÉ TABLE OF FUNCTIONS HAVING A FINITE NUMBER OF ESSENTIAL SINGULARITIES
}

\section{Albert Edrei}

Let $f(z)$ be regular at the origin and let it be single-valued and regular except for poles and $s+1<+\infty$ essential singularities $\alpha_{0}, \alpha_{1}, \alpha_{2}, \cdots, \alpha_{\mathrm{s}}$. The $\alpha$ 's may be limit-points of poles and $\alpha_{0}=\infty$ is permissible. Assume that $\alpha_{k}$ is of finite order $\lambda_{k}$ and let $\Lambda=\Sigma \lambda_{k}$.

The author obtains a convergence theorem for the Padé table of $\sum a_{1} z^{j}=f(z)$. The simplest consequence of his result may be stated as follows: if $\Lambda<2$, the diagonal approximants of the Padé table converge almost everywhere in the plane.

Introduction. Let

$$
a_{0}+a_{1} z+a_{2} z^{2}+\cdots=f(z) \quad\left(a_{0} \neq 0\right),
$$

have radius of convergence $\rho_{0}>0$. It is well known that given two integers $m \geqq 0, n \geqq 0$, it is always possible to find two polynomials

$$
\begin{aligned}
& Q_{m n}(z)=q_{0}+q_{1} z+\cdots+q_{n} z^{n}, \\
& P_{m n}(z)=p_{0}+p_{1} z+\cdots+p_{m} z^{m},
\end{aligned}
$$

such that

$$
Q_{m n}(z) \not \equiv 0
$$

and

$$
f(z) Q_{m n}(z)-P_{m n}(z)=\sum_{j=m+n+1}^{x} p_{j} z^{j}
$$

Although $P$ and $Q$ are not unique, the rational function

$$
R_{m n}(z)=\frac{P_{m n}(z)}{Q_{m n}(z)}
$$

does not depend on the particular choice of the polynomials $P$ and $Q$ which satisfy (4) and (5) [6; pp. 235-237]. We place $R_{m n}(z)$ in the $n$th row and $m$ th column of an infinite matrix. The resulting array is, by definition, the Padé table of $f(z)$. 
We propose to study the convergence of infinite sequences of "approximants" defined as follows: with each $k=1,2,3, \cdots$, we associate an ordered pair $(m, n)$

$$
m=m(k) \quad(m \geqq 0), n=n(k) \quad(n \geqq 0),
$$

and examine the behavior of the "error"

$$
f(z)-R_{m, n}(z)=\Delta_{m n}(z)=\Delta_{k}(z),
$$

as $k \rightarrow \infty$. The functions $m(k)$ and $n(k)$ in (7) enable us to simplify our notation and, from this point on, we write

$$
P_{m}(z), Q_{n}(z),
$$

instead of

$$
P_{m n}(z), Q_{m n}(z)
$$

Our results concern the class of

Quasi-meromorphic functions of finite order. We say that $f(z)$ is quasi-meromorphic, of order $\Lambda$, if

(i) $f(z)$ is single-valued; its only singularities are poles and essential singularities

$$
\alpha_{0}, \alpha_{1}, \cdots, \alpha_{s} \quad(s<+\infty),
$$

which may be limit-points of poles;

(ii) one of the $\alpha$ 's may be $\infty$;

(iii) the order of $\alpha_{j}$ is $\lambda_{j}$ and

$$
\lambda_{0}+\lambda_{1}+\lambda_{2}+\cdots+\lambda_{s}=\Lambda<+\infty .
$$

THEOREM 1. Let $f(z)$ be quasi-meromorphic of order $\Lambda$. Assume that $m(k)>0$ and $n(k)>0$ are integers such that

$$
\chi^{k^{\beta}<m+n} \quad(k=1,2,3, \cdots ; \chi>0)
$$

for some fixed $\chi$ and

$$
2 \beta>\Lambda .
$$

Assume also that, as $k \rightarrow \infty$, 


$$
m=o(n \log n), \quad n=o(m \log m)
$$

Then, given $\rho>0$ and $\delta>0$, it is possible to find a measurable set $\Omega=\Omega(\rho, \delta)$ such that

$$
\text { meas } \Omega<\delta \text {, }
$$

and such that

$$
R_{m n}(z) \rightarrow f(z) \quad(k \rightarrow+\infty),
$$

uniformly for all $z$ restricted by the conditions

$$
|z| \leqq \rho, \quad z \notin \Omega \text {. }
$$

As an immediate consequence, we obtain

Corollary 1. If $\Lambda<2$, then

$$
R_{n n}(z) \rightarrow f(z) \quad(n \rightarrow \infty)
$$

for almost all $z$.

The connection between the uniform convergence in (14) and the pointwise convergence in (16) is readily established by Egoroff's theorem. An appeal to the latter result does not simplify our proofs and we shall therefore not concern ourselves with this aspect of the question.

The introduction, in Theorem 1, of the exceptional set $\Omega$ cannot be avoided unless the class of functions under consideration is severely restricted [1], [4].

We study this situation in the following Theorem 2. For simplicity we confine our attention to sequences of diagonal elements of the table.

THEOREM 2. Let $f(z)$ be quasi-meromorphic, of order $\Lambda \leq+\infty$.

I. Consider the Hankel determinants

(17) $\quad A_{n}=\left|\begin{array}{lllll}a_{n} & a_{n-1} & a_{n-2} & \ldots & a_{1} \\ a_{n+1} & a_{n} & a_{n-1} & \ldots & a_{2} \\ \cdots & \cdots & \cdots & \cdots & \ldots \\ a_{2 n-1} & a_{2 n-2} & a_{2 n-3} & \cdots & a_{n}\end{array}\right|(n=1,2,3, \cdots)$, 
and let $A_{n} \neq 0$ for all $n$ belonging to some infinite sequence $\mathcal{N}$ of positive, strictly increasing integers.

Assume that, in some neighborhood of the origin, the approximants $R_{n n}(z)(n \in \mathcal{N})$ have no poles.

Then

$$
\limsup _{\substack{n \rightarrow \infty \\ n \in \mathcal{N}}}\left|\frac{A_{n+1}}{A_{n}}\right|^{1 / n \log n} \leqq e^{-1 / \Lambda}
$$

II. Assume that the disk

$$
\left|z-z_{0}\right|<\eta \quad(\eta>0)
$$

contains no singularities of $f(z)$ and no poles of $R_{n n}(z)$.

Then, given $\eta^{\prime}\left(0<\eta^{\prime}<\eta\right)$ and $\delta>0$ we have

$$
\left|f(z)-R_{n n}(z)\right| \leqq \exp \left(-\frac{n \log n}{\Lambda+\delta}\right)
$$

for all $z$ and $n$ satisfying the conditions

$$
\left|z-z_{0}\right| \leqq \eta^{\prime}, \quad n \geqq N_{0}\left(\eta^{\prime}, \delta\right), \quad A_{n} \neq 0 .
$$

The method which leads to Theorems 1 and 2 may be applied to situations significantly more general than the one considered in this paper.

Functions with singularities of transfinite measure zero. We say that the singularities of $f(z)$ have transfinite measure zero if $f(z)$ satisfies the two following conditions.

I. The analytic function $f(z)$ is single-valued and regular at the origin and throughout the complement $C E$ of a closed set $E$ of the extended $z$-plane. The origin lies in $C E$.

II. Consider, in the plane of the complex variable $\zeta$, the image $\mathscr{E}$ of $E$ given by the inversion

$$
\zeta=z^{-1}
$$

Assume

$$
\tau(\mathscr{E})=0
$$

where $\tau(\mathscr{E})$ is the transfinite diameter [5; pp. 268-273] of $\mathscr{E}$. 
We state without proof the following analogue of assertion I of Theorem 2.

THEOREM 3. Let the singularities of $f(z)$ have transfinite measure zero.

Let $A_{n}$ and $\mathcal{N}$ have the same meaning as in Theorem 2. Assume that, in some neighborhood of the origin, the approximants $R_{n n}(z)$ $(n \in \mathcal{N})$ have no poles.

Then

$$
\lim _{\substack{n \rightarrow \infty \\ n \in \mathcal{N}}}\left|\frac{A_{n+1}}{A_{n}}\right|^{1 / n}=0
$$

An analogous extension of assertion II of Theorem 2 is also possible. We do not state it here because, in its present form, our proof introduces restrictions which are more complex than those given by (19).

The analogue of Theorem 1, for functions with singularities of transfinite measure zero, differs little from a recent result of Pommerenke [8]; it is consequently omitted from this note.

If $f(z)$ has singularities of transfinite measure zero, a classical result of Pólya [7; pp. 688-689] asserts that

$$
\lim _{n \rightarrow \infty}\left|A_{n}\right|^{1 / n^{2}}=0
$$

For the class of quasi-meromorphic functions of order $\Lambda$, I have shown [3; pp. 36-49] that

$$
\limsup _{n \rightarrow \infty}\left|A_{n}\right|^{1 / n^{2} \log n} \leqq e^{-1 / \Lambda}
$$

and that this relation is "best possible".

From (21) and (22) we deduce, respectively,

$$
\liminf _{n \rightarrow \infty}\left|A_{n+1} / A_{n}\right|^{1 / n}=0,
$$

and

$$
\liminf _{n \rightarrow \infty}\left|A_{n+1} / A_{n}\right|^{1 / 2 n \log n} \leqq e^{-1 / \Lambda} .
$$

The corresponding relations with lim inf replaced by lim sup require, in addition, that the sequence $\left\{\left|A_{n}\right|\right\}_{n}$ be decreasing with a regularity that has little analytic significance. 
On the other hand (18) and (20) show that, unless the determinants $A_{n}$ display this type of regularity, the sequence of diagonal approximants $\left\{R_{n n}(z)\right\}_{n}$ will have poles accumulating at the origin. Following Chisholm [2], we say that such poles are "spurious"; they are accidents of the Padé method and do not reflect the presence of nearby singularities of $f(z)$. To eliminate spurious poles, we would have to impose severe and unnatural conditions: within the class of quasi-meromorphic functions of order $\Lambda$,

$$
\left.\limsup _{n \rightarrow \infty}\left|A_{n+1}\right| A_{n}\right|^{1 / n \log n} \leqq e^{-1 / \Lambda}
$$

represents as serious a restriction as would be the requirement that, within the class of entire functions of finite order (for which $\lim \sup _{n \rightarrow \infty}\left|a_{n}\right|^{1 / n \log n}<1$ ), we only consider those for which

$$
\underset{n \rightarrow \infty}{\limsup }\left|a_{n+1} / a_{n}\right|^{1 / \log n}<1 .
$$

Focusing our attention on the class of quasi-meromorphic functions of order $\Lambda<2$, we see that the perturbations introduced by spurious poles are to some extent compensated by the fact that the convergence of the approximants is unaffected by the radius of convergence of (1) (or the radius of meromorphy of $f(z)$ ): in an obvious sense, the sequence

$$
\left\{R_{n n}(z)\right\}_{n}
$$

of diagonal approximants "overconverges" almost everywhere in the plane.

We conclude this introduction by some remarks about the notations $K, K_{0}, N_{0}$, which we use systematically. We always assume that $K>1$; the value of $K$ may depend on several parameters and is not necessarily the same one each time it occurs. The quantities $K_{0}, N_{0}$ denote positive integers with the same indeterminate character as $K$. They always appear as conditions such as $k \geqq K_{0}, n \geqq N_{0}$, and restrict the validity of some relation to sufficiently large values of $k$ and $n$. Whenever they qualify expressions such as (19), involving the complex variable $z$, it is understood that the bounds $K_{0}$ or $N_{0}$ which they indicate hold uniformly for all $z$ under consideration.

\section{Integral representation of the error term} $\Delta_{m n}(z)$. Using the notation (9), we rewrite (5) as 
(2.1)

$$
f(z) Q_{n}(z)-P_{m}(z)=\sum_{j=m+n+1}^{\infty} p_{j} z^{j} \quad\left(p_{j}=p_{j}(k)\right) .
$$

Set

$$
a_{l}=0 \cdot \quad(l=-1,-2,-3, \cdots)
$$

consider the Hankel matrix

$$
\left(\begin{array}{ccccc}
a_{m+1} & a_{m} & a_{m-1} & \ldots & a_{m-n+1} \\
a_{m+2} & a_{m+1} & a_{m} & \ldots & a_{m-n+2} \\
\ldots & \ldots & \ldots & \ldots & \ldots \\
a_{m+n} & a_{m+n-1} & a_{m+n-2} & \ldots & a_{m}
\end{array}\right),
$$

and assume that its rank is $n$. We may then obtain a "solution" of (2.1) by defining

(2.4) $\quad Q_{n}(z)=\left|\begin{array}{ccccc}1 & z & z^{2} & \ldots & z^{n} \\ a_{m+1} & a_{m} & a_{m-1} & \ldots & a_{m-n+1} \\ a_{m+2} & a_{m+1} & a_{m} & \ldots & a_{m-n+2} \\ \ldots & \ldots & \ldots & \ldots & \ldots \\ a_{m+n} & a_{m+n-1} & a_{m+n-2} & \ldots & a_{m}\end{array}\right|$,

(2.5) $p_{j}=\left|\begin{array}{lllll}a_{j} & a_{j-1} & a_{j-2} & \cdots & a_{j-n} \\ a_{m+1} & a_{m} & a_{m-1} & \cdots & a_{m-n+1} \\ a_{m+2} & a_{m+1} & a_{m} & \cdots & a_{m-n+2} \\ \cdots & \cdots & \cdots & \cdots & \cdots \\ a_{m+n} & a_{m+n-1} & a_{m+n-2} & \cdots & a_{n}\end{array}\right|(j=0,1,2,3, \cdots)$,

and

$$
P_{m}(z)=\sum_{j=0}^{m} p_{j} z^{j}
$$

Whenever the rank of (2.3) is $n$, the polynomials $P_{m}$ and $Q_{n}$ will be defined by (2.6) and (2.4). Then

$$
f(z) Q_{n}(z)-P_{m}(z)=(-1)^{n} A_{m+1}^{(n+1)} z^{m+n+1}+z^{m+n+2} \mathscr{S}(z),
$$


where $\mathscr{S}(z)$ is a series of nonnegative powers of $z$ and $A_{m+1}^{(n+1)}$ is the Hankel determinant of the matrix obtained by adding to (2.3), as last row

$$
a_{m+n+1}, a_{m+n}, \cdots a_{m+1} .
$$

If the rank of (2.3) is $<n,(2.4)$ becomes $Q_{n}(z) \equiv 0$; it violates our condition (4) and hence cannot be used. For sake of definiteness we shall then select, for $P_{m}$ and $Q_{n}$, some specific solution of (2.1) with

$$
\text { degree } P_{m} \leqq m, \quad \text { degree } Q_{n} \leqq n, \quad Q_{n}(z) \not \equiv 0 .
$$

A closer characterization of these polynomials is unnecessary.

Denote by

$$
V_{n}(z)=V_{n m}(z)
$$

a polynomial such that

$$
\text { degree } V_{n} \leqq n, \quad V_{n}(z) \not \equiv 0,
$$

otherwise arbitrary.

From (2.1) we deduce that

$$
\frac{f(z) Q_{n}(z) V_{n}(z)-P_{m}(z) V_{n}(z)}{z^{m+n+1}}=F_{n}(z)
$$

is holomorphic for $|z|<\rho_{0}$ and hence by Cauchy's theorem

(2.11) $F_{n}(z)=\frac{1}{2 \pi i} \oint \frac{f(\zeta) Q_{n}(\zeta) V_{n}(\zeta)}{\zeta^{m+n+1}(\zeta-z)} d \zeta-\frac{1}{2 \pi i} \oint \frac{P_{m}(\zeta) V_{n}(\zeta)}{\zeta^{m+n+1}(\zeta-z)} d \zeta$,

where $|z|<\rho_{0}$ and the contour of integration is a circumference

$$
\zeta=r_{0} e^{i \omega} \quad\left(0 \leqq \omega<2 \pi,|z|<r_{0}<\rho_{0}\right) .
$$

Since $P_{m}(z) V_{n}(z)$ is of degree $\leqq m+n$, the second integral in (2.11) is zero and hence we obtain the fundamental relation

$$
\begin{aligned}
f(z)-\frac{P_{m}(z)}{Q_{n}(z)} & =\Delta_{m n}(z) \\
& =\frac{z^{m+n+1}}{Q_{n}(z) V_{n}(z)} \frac{1}{2 \pi i} \oint \frac{f(\zeta) Q_{n}(\zeta) V_{n}(\zeta)}{\zeta^{m+n+1}(\zeta-z)} d \zeta
\end{aligned}
$$


We note that (2.1) and (2.11) also imply

$$
F_{n}(0)=p_{m+n+1} V_{n}(0)=\frac{1}{2 \pi i} \oint \frac{f(\zeta) Q_{n}(\zeta) V_{n}(\zeta)}{\zeta^{m+n+2}} d \zeta
$$

We shall use the above relation in the special case

$$
m=n, \quad A_{n} \neq 0, \quad V_{n}(0) \neq 0 .
$$

Since

$$
A_{n}=A_{n}^{(n)}=Q_{n}(0),
$$

we may rewrite (2.14) in the equivalent form

$$
(-1)^{n} \frac{A_{n+1}}{A_{n}}=\frac{1}{2 \pi i V_{n}(0)} \oint \frac{f(\zeta) V_{n}(\zeta)\left\{Q_{n}(\zeta) / Q_{n}(0)\right\}}{\zeta^{2 n+2}} d \zeta
$$

We also note that (2.7) (with $m=n$ ) leads at once to

$$
Q_{n-1}(z) P_{n}(z)-Q_{n}(z) P_{n-1}(z)=(-1)^{n-1} A_{n}^{2} z^{2 n-1} \text {. }
$$

Hence, if $A_{n} \neq 0, P_{n}(z)$ and $Q_{n}(z)$ have no common zeros and the poles of $R_{n n}(z)$ coincide exactly with the zeros of $Q_{n}(z)$.

3. Factors of a quasi-meromorphic function. From our definitions it follows that $f(z)$, in Theorem 1 , may be represented in the form

$$
f(z)=h_{0}(z) h_{1}\left(\frac{1}{z-\alpha_{1}}\right) h_{2}\left(\frac{1}{z-\alpha_{2}}\right) \cdots h_{s}\left(\frac{1}{z-\alpha_{s}}\right) \quad(s<+\infty)
$$

where

$$
h_{\nu}(t) \quad(\nu=0,1,2, \cdots, s)
$$

is a meromorphic function of $t$, of order $\lambda_{\nu}<+\infty$. Meromorphic functions correspond to the special case $s=0$.

The possibility of a decomposition such as (3.1) and the uniqueness of the orders $\lambda_{\nu}(\nu=0,1, \cdots, s)$ are readily deducible from the definition of order of an essential singularity.

This aspect of the question need not concern us here and we shall be content with the naive view that Theorem 1 is applicable to all functions representable in the form (3.1). 
It is obvious that a finite number of zeros and poles can always be transferred from one factor $h$ to another. This remark leads us to a preliminary clarification of what we mean by zeros and poles of $h_{\nu}(t)$.

Select $\gamma_{0}$ so as to satisfy the conditions

$$
0<2 \gamma_{0}<\min \left(\left|\alpha_{1}\right|,\left|\alpha_{2}\right|, \cdots,\left|\alpha_{s}\right|, 1\right),
$$

and

$$
2 \gamma_{0}<\min _{\nu \neq \mu}\left(\left|\alpha_{\nu}-\alpha_{\mu}\right|\right) \quad(1 \leqq \nu \leqq s, 1 \leqq \mu \leqq s) .
$$

The poles of $f(z)$ which lie in the region

$$
0<\left|z-\alpha_{\nu}\right|<\gamma_{0}
$$

will be denoted by

$$
b_{1}^{(\nu)}, b_{2}^{(\nu)}, b_{3}^{(\nu)}, \cdots
$$

and arranged so that

$$
\gamma_{0}>\left|b_{1}^{(\nu)}-\alpha_{\nu}\right| \geqq\left|b_{2}^{(\nu)}-\alpha_{\nu}\right| \geqq\left|b_{3}^{(\nu)}-\alpha_{\nu}\right| \geqq \cdots .
$$

The "poles of $h_{v}(t)$ " are, by definition, the quantities

$$
t_{1}^{(\nu)}, t_{2}^{(\nu)}, t_{3}^{(\nu)}, \cdots
$$

deduced from (3.6) by the transformation

$$
t_{j}^{(\nu)}=\frac{1}{b_{j}^{(\nu)}-\alpha_{\nu}} \quad(j=1,2,3, \cdots) .
$$

These operations are performed for all $\nu=1,2, \cdots, s$. The poles of $f(z)$ which do not belong to one of the sequences $\left\{b_{j}^{(\nu)}\right\}_{j}(\nu=1,2, \cdots, s)$, have no finite point of accumulation. We shall denote them by $b_{j}$ and arrange them in a sequence

$$
b_{1}, b_{2}, b_{3}, \cdots
$$

so that

$$
0<\rho_{0} \leqq\left|b_{1}\right| \leqq\left|b_{2}\right| \leqq\left|b_{3}\right| \leqq \cdots
$$


We also include, in (3.10), elements such as $\alpha_{\nu}$ (repeated a suitable number of times). They enable us to take into account factors $\left(z-\alpha_{\nu}\right)^{-\beta}(\beta>0)$ which may be present in the decomposition (3.1). The sequence (3.10) is by definition the sequence of poles of $h_{0}(z)$.

In view of the conditions (3.4), (3.5), and of the regularity of $f(z)$ at the origin, every pole of $f(z)$ belongs to one and only one of the factors $h_{\nu}$ in (3.1).

The zeros of $f(z)$ (which do not explicitly appear in our proofs) are to be distributed in exactly the same way among the $s+1$ functions $h_{\nu}(t)$. The assumption $a_{0} \neq 0$ implies the regularity of $1 / f(z)$ at the origin. Hence there is complete symmetry between the functions $f(z)$ and $1 / f(z)$. Both have the same essential singularities $\alpha_{\nu}$ with the same orders $\lambda_{\nu}(\nu=0,1,2, \cdots, s)$.

\section{Estimates from the theory of meromorphic function.} and let

Lemma 1. Let $h(z)(h(0) \neq \infty)$ be meromorphic, of finite order $\lambda$

$$
b_{1}, b_{2}, \cdots, b_{j}, \cdots \quad\left(0<\left|b_{1}\right| \leqq\left|b_{2}\right| \leqq\left|b_{3}\right| \leqq \cdots\right)
$$

be the sequence of its poles.

Take $\sigma>\lambda$. Then, if $n$ is large enough, the function $h(z)$ has fewer than $n$ poles in the disk

$$
|z| \leqq n^{1 / \sigma}
$$

The function

$$
g_{n}(z)=h(z) \prod_{\left|b_{j}\right| \leq n}\left(1-\frac{z}{b_{j}}\right),
$$

which is regular in the disk (4.1), satisfies the inequality

$$
\left|g_{n}(z)\right| \leqq e^{n} \quad\left(|z| \leqq \frac{1}{2} n^{1 / \sigma}\right) .
$$

Proof. Denote as usual by $n(x, \infty)[9 ;$ p. 284b] the number of poles of $h(z)$ in $|z| \leqq x$. By the elements of the theory

$$
n(x, \infty)<x^{\sigma^{\prime}} \quad\left(\lambda<\sigma^{\prime}<\sigma ; x>x_{0}\left(\sigma^{\prime}\right)\right) .
$$

Hence there are, in the disk (4.1), fewer than 


$$
n^{\sigma^{\prime} / \sigma}<n \quad\left(n \geqq N_{0}\right)
$$

poles of $h(z)$.

From the Poisson-Jensen-Nevanlinna identity [9; p. 129] we deduce the well-known inequality

$$
\log |h(z)| \leqq \frac{x+|z|}{x-|z|} m(x, h)+\sum_{\left|b_{j}\right| \leqq x} \log \left(\frac{2 x}{\left|z-b_{j}\right|}\right)
$$

where $|z|<x$ and $m(x, h)$ is Nevanlinna's mean. Applying (4.6) with

$$
x=n^{1 / \sigma}, \quad|z| \leqq \frac{1}{2} n^{1 / \sigma},
$$

we find

$$
\log \left|g_{n}(z)\right| \leqq 3 m\left(n^{1 / \sigma}, h\right)+n\left(n^{1 / \sigma}, \infty\right) \log 2+N\left(n^{1 / \sigma}, \infty\right)
$$

where $N(x, \infty)$ has the meaning assigned to it in Nevanlinna's theory.

The inequality (4.3) follows immediately from (4.7) and the wellknown behavior of $m(x, h), n(x, \infty), N(x, \infty)$ associated with functions of finite order.

5. Construction of the polynomials $V_{n}(z)$ and of the contours $\mathscr{C}_{n}$. Choose $\eta>0$ such that

$$
\sigma_{\nu}=\lambda_{\nu}+\eta \quad(\nu=0,1,2, \cdots, s)
$$

satisfy the condition

$$
\sum_{\nu=0}^{s} \sigma_{\nu}=\Sigma<2 \beta
$$

This is possible by (11).

Put

$$
n_{\nu}=\left[\frac{\sigma_{\nu} n}{\Sigma}\right] \quad(\nu=0,1,2, \cdots, s)
$$

which imply

$$
n_{\nu} \sim \frac{\sigma_{\nu} n}{\Sigma} \quad(\nu=0,1,2, \cdots, s ; n \rightarrow \infty)
$$

and 


$$
\sum_{\nu=0}^{s} n_{\nu} \leqq n
$$

The contour $\mathscr{C}_{n}\left(n \geqq N_{0}\right)$ is formed by $s+1$ circumferences:

(5.6) $\mathscr{C}_{n}^{(0)}=\left\{\zeta: \zeta=\frac{1}{2} e^{i \omega} n_{0}^{1 / \sigma_{0}} \quad(0 \leqq \omega<2 \pi)\right\}$,

$(5.7) \mathscr{C}_{n}^{(\nu)}=\left\{\zeta: \zeta=2 e^{-i \omega} n_{\nu}^{-1 / \sigma_{\nu}}+\alpha_{\nu} \quad(0 \leqq \omega<2 \pi)\right\} \quad(\nu=1,2, \cdots, s)$.

We introduce, for later use, the compact set

$$
\mathscr{D}_{n}=\left\{z:|z| \leqq \frac{1}{2} n_{0}^{1 / \sigma_{0}}\right\}-\bigcup_{\nu=1}^{s}\left\{z:\left|z-\alpha_{\nu}\right|<2 n_{\nu}^{-1 / \sigma_{\nu}}\right\},
$$

which is bounded by

$$
\mathscr{C}_{n}=\bigcup_{\nu=0}^{s} \mathscr{C}_{n}^{(\nu)}
$$

Consider now the points $b_{j}^{(\nu)}$ which appear in (3.6) and (3.7); let there be $l^{(\nu)}$ of them which satisfy the condition

$$
\left|b_{j}^{(\nu)}-\alpha_{\nu}\right| \geqq n_{\nu}^{-1 / \sigma_{\nu}}
$$

In view of Lemma 1

$$
l^{(\nu)}<n_{\nu}
$$

Let

$$
\begin{aligned}
V_{n \nu}(z) & =\left(z-\alpha_{\nu}\right)^{n_{\nu}-l^{(\nu)}} \prod_{j=1}^{(\nu)}\left(z-b_{j}^{(\nu)}\right) \\
& =\left(z-\alpha_{\nu}\right)^{n_{\nu}} \prod_{j=1}^{(\nu)} \frac{z-b_{j}^{(\nu)}}{z-\alpha_{\nu}}=\left(z-\alpha_{\nu}\right)^{n_{\nu}} W_{n \nu}(z) .
\end{aligned}
$$

We thus define $V_{n \nu}(z)(\nu=1,2, \cdots, s)$ and finally

$$
V_{n 0}(z)=\prod_{\left|b_{j}\right| \leq n_{o}^{\prime \sigma_{0}}}\left(1-\frac{z}{b_{j}}\right),
$$

where the $b_{j}$ are members of (3.10). By Lemma 1, the degree $l^{(0)}$ of $V_{n 0}(z)$ satisfies the condition

$$
l^{(0)}<n_{0} .
$$


Our polynomial $V_{n}(z)$ is now defined as

$$
V_{n}(z)=\prod_{\nu=0}^{s} V_{n \nu}(z)
$$

its degree is

$$
l^{(0)}+n_{1}+n_{2}+\cdots+n_{s}<n .
$$

In view of (3.1), (5.11), (5.12) and (5.14)

(5.16)f $(z) V_{n}(z)=h_{0}(z) V_{n 0}(z)\left\{\prod_{\nu=1}^{s} h_{\nu}\left(\frac{1}{z-\alpha_{\nu}}\right) W_{n \nu}(z)\right\} \prod_{\nu=1}^{s}\left(z-\alpha_{\nu}\right)^{n_{\nu}}$.

6. Estimates for $f(z) V_{n}(z)$ and $V_{n}(z)$. If $n \geqq N_{0}$, Lemma 1 shows that $h_{0}(z) V_{n 0}(z)$ is regular at all points of the disk $|z| \leqq \boldsymbol{n}_{0}^{1 / \sigma_{0}}$. Moreover

$$
\left|h_{0}(z) V_{n 0}(z)\right| \leqq e^{n_{0}} \quad\left(|z| \leqq \frac{n_{0}^{1 / \sigma_{0}}}{2}\right) .
$$

Similarly, the substitution

$$
t=\frac{1}{z-\alpha_{\nu}}
$$

transforms

$$
h_{\nu}\left(\frac{1}{z-\alpha_{\nu}}\right) W_{n \nu}(z)
$$

into

$$
h_{\nu}(t) \prod_{j=1}^{(\omega)}\left(1-\frac{t}{t_{j}^{(\nu)}}\right)
$$

Again, by Lemma 1, the product (6.2) is regular for

$$
\left|z-\alpha_{\nu}\right| \geqq n_{\nu}^{-1 / \sigma_{\nu}}
$$

and satisfies the inequality

$$
\left|h_{\nu}\left(\frac{1}{z-\alpha_{\nu}}\right) W_{n \nu}(z)\right| \leqq e^{n_{\nu}} \quad\left(\left|z-\alpha_{\nu}\right| \geqq 2 n_{\nu}^{-1 / \sigma_{\nu}}\right) .
$$


Lemma 2. If $n$ is large enough

$$
f(z) V_{n}(z)
$$

is regular at all points of $\mathscr{D}_{n}$.

Moreover

$$
\left|f(\zeta) V_{n}(\zeta)\right| \leqq e^{n}(\tilde{K})^{n} \exp \left(-\frac{n_{\nu} \log n_{\nu}}{\sigma_{\nu}}\right) \quad\left(\zeta \in \mathscr{C}_{n}^{(\nu)} \nu \neq 0\right)
$$

with

$$
\tilde{K}=\left|\alpha_{1}\right|+\left|\alpha_{2}\right|+\cdots+\left|\alpha_{s}\right|+2
$$

and

$$
\left|f(\zeta) V_{n}(\zeta)\right| \leqq(2 e)^{n}|\zeta|^{n_{1}+n_{2}+\cdots+n_{\varsigma}} \quad\left(\zeta \in \mathscr{C}_{n}^{(0)}\right) .
$$

Proof. The regularity of the product (6.4) in $\mathscr{D}_{n}$ is an immediate consequence of the decomposition (5.16) and of the regularity of each factor (6.2) and of $h_{0}(z) V_{n 0}(z)$.

The estimates (6.5) and (6.6) are obvious consequences of (5.16), of the estimates (6.1) and (6.3), and of the definitions of the contours $\mathscr{C}_{n}^{(\nu)}$.

This proves Lemma 2. We also need the lower bound for $V_{n}(z)$ contained in

Lemma 3. Consider the point sets

$$
D(\rho, \gamma)=\{z:|z| \leqq \rho\}-\bigcup_{\nu=1}^{s}\left\{z:\left|z-\alpha_{\nu}\right|<\gamma\right\}
$$

and

$$
D\left(2 \rho, \frac{\gamma}{2}\right)=\{z:|z| \leqq 2 \rho\}-\bigcup_{\nu=1}^{s}\left\{z:\left|z-\alpha_{\nu}\right|<\frac{\gamma}{2}\right\},
$$

where

$$
\rho \geqq 1+\max _{1 \leqq \nu \leqq s}\left|\alpha_{\nu}\right|, \quad 0<\gamma<\gamma_{0},
$$

and $\gamma_{0}$ is the constant in (3.3) and (3.4).

Let there be $\mathscr{S}=\mathscr{S}(2 \rho, \gamma / 2)$ poles of $f(z)$ in $D(2 \rho, \gamma / 2)$; rename them 


$$
c_{1}, c_{2}, \cdots, c_{\mathscr{S}}
$$

and let

$$
H_{n}=\bigcup_{j=1}^{\varphi}\left\{z:\left|z-c_{j}\right|<e^{-n}\right\} .
$$

Then, the condition

$$
z \in D(\rho, \gamma)-H_{n}
$$

implies

$$
\left|V_{n}(z)\right| \geqq(\gamma / 2 \rho)^{n} e^{-\varphi_{n}}
$$

Proof. An inspection of (5.14), (5.11) and (5.12), shows that each one of the linear factors $\left(z-c_{j}\right)$ or $\left(1-z / c_{j}\right)$ which may appear in $V_{n}(z)$ has a modulus $\geqq e^{-n} / 2 \rho$, provided $z \notin H_{n}$. For $z \in D(\rho, \gamma)$, all other factors have a modulus $\geqq \gamma / 2 \rho$. The Lemma is now obvious.

\section{Proof of assertion I of Theorem 2. Consider}

$$
R_{n n}(z)=\frac{P_{n}(z)}{Q_{n}(z)}
$$

where $q=q(n) \leqq n$ is the exact degree of $Q_{n}(z)$.

If $n \in \mathcal{N}$, we have

$$
A_{n}=Q_{n}(0) \neq 0
$$

and hence, if $Q_{n}(z)$ has zeros $z_{n j}$,

$$
\frac{Q_{n}(z)}{Q_{n}(0)}=\left(1-\frac{z}{z_{n 1}}\right)\left(1-\frac{z}{z_{n 2}}\right) \cdots\left(1-\frac{z}{z_{n q}}\right)
$$

In view of the remark at the end of $\$ 2$, the poles of $R_{n n}(z)$ coincide exactly with the zeros of $Q_{n}(z)$ so that by one of the assumptions of Theorem 2, we have

$$
\left|\frac{Q_{n}(z)}{Q_{n}(0)}\right| \leqq\left(1+\frac{|z|}{\delta}\right)^{n} \quad(\delta>0, n \in \mathcal{N})
$$

where $\delta$ is suitably small and fixed.

By (7.1), (2.16), Lemma 2, and the elements of contour integration, 


$$
(-1)^{n} \frac{A_{n+1}}{A_{n}}=\frac{1}{V_{n}(0)} \sum_{\nu=0}^{s} I_{n v}
$$

with

$$
I_{n \nu}=\frac{1}{2 \pi i} \int_{\mathscr{C}_{n}^{(\nu)}} \frac{f(\zeta) V_{n}(\zeta)\left\{Q_{n}(\zeta) / Q_{n}(0)\right\}}{\zeta^{2 n+2}} d \zeta
$$

By (7.2) and (6.5)

$$
\begin{gathered}
\left|I_{n \nu}\right| \leqq K^{n} \exp \left(-\frac{n_{\nu} \log n_{\nu}}{\sigma_{\nu}}\right)\left(1+\frac{\tilde{K}}{\delta}\right)^{n} \gamma_{0}^{-2 n-1} \\
\left(n \geqq N_{0} ; \nu=1,2, \cdots, s\right),
\end{gathered}
$$

where $\gamma_{0}$ satisfies (3.3).

Similarly, using (6.6) instead of (6.5), we find

$$
\left|I_{n 0}\right| \leqq K^{n} \exp \left(-\frac{n_{0} \log n_{0}}{\sigma_{0}}\right)\left\{\frac{2}{n_{0}^{1 / \sigma_{0}}}+\frac{1}{\delta}\right\}^{n} \quad\left(n \geqq N_{0}\right) .
$$

By Lemma 3,

$$
\frac{1}{\left|V_{n}(0)\right|} \leqq K^{n}
$$

We now use (7.5), (7.6) and (7.7) in (7.3) and obtain

$$
\left|\frac{A_{n+1}}{A_{n}}\right| \leqq K^{n} \sum_{\nu=0}^{s} \exp \left(-\frac{n_{\nu} \log n_{\nu}}{\sigma_{\nu}}\right) \quad\left(n \in \mathcal{N}, n \geqq N_{0}\right) .
$$

In view of (5.4), this leads to

$$
\limsup _{\substack{n \rightarrow \infty \\ n \in \mathcal{N}}}\left|\frac{A_{n+1}}{A_{n}}\right|^{1 / n \log n} \leqq e^{-1 / \Sigma} .
$$

Since

$$
\Sigma=\Lambda+(s+1) \eta \quad(\eta>0),
$$

and $\eta$ is arbitrary, (18) follows from (7.8) and assertion I of Theorem 2 is proved. 


\section{A consequence of the Boutroux-Cartan lemma.}

LemMA 4. Let

$$
Q_{n}(z)=\kappa_{n}\left(z-z_{n 1}\right)\left(z-z_{n 2}\right) \cdots\left(z-z_{n q}\right) \quad\left(\kappa_{n} \neq 0\right)
$$

be a polynomial of degree $q \leqq n$.

Then, given $\epsilon>0$ and $\rho \geqq \epsilon$, it is possible to find a set $G_{n}$, depending only on $\epsilon, \rho$, and on the zeros of $Q_{n}(z)$, formed by the union of no more than $n$ disks with sum of radii $\leqq 2 e \epsilon$ and such that

$$
|z| \leqq \rho, \quad z \notin G_{n}
$$

imply

$$
\left|\frac{Q_{n}(\zeta)}{Q_{n}(z)}\right| \leqq\left(\frac{\lfloor\zeta \mid+2 \rho}{\epsilon}\right)^{n}
$$

Each one of the disks of $G_{n}$ contains one or more zeros of $Q_{n}(z)$.

Proof. Let

$$
z_{n \nu} \quad(\nu=1,2, \cdots, l)
$$

be all those zeros of $Q_{n}(z)$ which lie in $|z| \leqq 2 \rho$.

In view of $(8.2)$

$$
\left|Q_{n}(z)\right| \geqq\left|\kappa_{n}\right| \prod_{\nu=l+1}^{q}\left|\frac{z_{n v}}{2}\right| \prod_{\nu=1}^{l}\left|z-z_{n \nu}\right|,
$$

and by the Boutroux-Cartan lemma $[10 ;$ p. 60]

$$
\left|Q_{n}(z)\right| \geqq\left|\kappa_{n}\right| \epsilon^{\prime} 2^{1-q} \prod_{\nu=1+1}^{q}\left|z_{n \nu}\right| \quad\left(z \notin G_{n}\right) .
$$

A classical proof of the Boutroux-Cartan lemma [10] shows that each exceptional disk of $G_{n}$ must contain zeros of $Q_{n}(z)$.

The upper bound

$$
\left|Q_{n}(\zeta)\right| \leqq\left|\kappa_{n}\right|(|\zeta|+2 \rho)^{l} \prod_{\nu=l+1}^{q}\left(1+\frac{|\zeta|}{2 \rho}\right)\left|z_{n \nu}\right|
$$

is obvious so that (8.3) follows immediately from (8.4) and (8.5). 
9. Analytic continuation of the error term. We start from (2.13) with $|z|<r_{0}<\rho_{0}$ and with the contour of integration (2.12).

By Lemma 2 and the elements of contour integration

$$
f(z)-R_{m n}(z)=\frac{z^{m+n+1}}{Q_{n}(z) V_{n}(z)} \sum_{\nu=0}^{s} J_{n v} \quad\left(n \geqq N_{0}\right)
$$

where

$$
J_{n \nu}=\frac{1}{2 \pi i} \int_{\mathscr{C}_{n}^{(\nu)}} \frac{f(\zeta) V_{n}(\zeta) Q_{n}(\zeta)}{\zeta^{m+n+1}(\zeta-z)} d \zeta .
$$

An inspection of (6.7) and (5.8) shows that, if $n$ is large enough, the identity (9.1) is valid for all points of $D(\rho, \gamma)$ other than the zeros of $V_{n}(z) Q_{n}(z)$.

10. Proof of assertion II of Theorem 2. We take $m=n$ in (9.1) and (9.2).

By assumption

$$
\Gamma_{0}=\left\{z:\left|z-z_{0}\right|<\eta\right\}
$$

contains no poles of $R_{n n}(z)$ and, since $A_{n} \neq 0$, it also contains no zeros of $Q_{n}(z)$.

We use Lemma 4 with

$$
4 \epsilon e<\eta-\eta^{\prime} .
$$

Then, the exceptional disks do not intersect

$$
\Gamma_{1}=\left\{z:\left|z-z_{0}\right| \leqq \eta^{\prime}\right\}
$$

and consequently

$$
\left|\frac{Q_{n}(\zeta)}{Q_{n}(z)}\right| \leqq\left(\frac{\lfloor\zeta \mid+2 \rho}{\epsilon}\right)^{n} \quad\left(z \in \Gamma_{1}\right) .
$$

Take in Lemma 3

$$
\gamma<\frac{\eta-\eta^{\prime}}{2}
$$

$\rho$ large enough to imply 


$$
\Gamma_{0} \subset\{z:|z| \leqq \rho\}
$$

and $n$ large enough to imply

$$
\Gamma_{1} \subset D(\rho, \gamma)-H_{n}
$$

Then

$$
\left|\frac{1}{V_{n}(z)}\right| \leqq K^{n} \quad\left(K>1, z \in \Gamma_{1}\right)
$$

for some suitable value of $K$.

From (9.2), (10.1) and (10.2) we deduce

$$
\left|\frac{J_{n \nu}}{Q_{n}(z) V_{n}(z)}\right| \leqq\left(\frac{K}{\epsilon}\right)^{n} \frac{1}{\gamma_{0}\left(\eta-\eta^{\prime}\right)} \max _{\zeta}\left\{\left(1+\frac{2 \rho}{|\zeta|}\right)^{n}\left|\frac{f(\zeta) V_{n}(\zeta)}{\zeta^{n}}\right|\right\}
$$

where

$$
\zeta \in \mathscr{C}_{n}^{(\nu)}, \quad \nu=0,1,2, \cdots, s, \quad n \geqq N_{0} .
$$

Using these estimates, (6.5) and (6.6) in (9.1), we find

(10.3) $\left|f(z)-R_{n n}(z)\right| \leqq K^{n} \sum_{\nu=0}^{s} \exp \left(-\frac{n_{\nu} \log n_{\nu}}{\sigma_{\nu}}\right) \quad\left(z \in \Gamma_{1}, n \geqq N_{0}\right)$.

In view of (5.4), assertion II of Theorem 2 is an elementary consequence of (10.3).

11. Proof of Theorem 1. We first restrict our arguments to the approximants on the side of the diagonal characterized by

$$
m(k) \geqq n(k),
$$

and assume that there are infinitely many approximants satisfying the above condition.

By (10), (12) and (11.1) we obtain

$$
\beta \log k<\log n+o(\log n) \quad(k \rightarrow \infty),
$$

and therefore $n \rightarrow \infty$ as $k \rightarrow \infty$.

We now use (5.2) to select $\xi>0$, small enough to imply 


$$
(\Sigma+3 \xi)<\frac{2 \beta}{1+\xi}
$$

and deduce from (11.2)

$$
(\Sigma+3 \xi) \log \left(k^{(1 / 2)+(\xi / 2)}\right)<\log n+o(\log n) \quad(k \rightarrow \infty) .
$$

Let $\rho$ and $\delta$ be the given quantities in the statement of Theorem 1. We increase, if necessary, the value of $\rho$ so that the first inequality in (6.9) be satisfied; this does not affect the generality of Theorem 1. With $\rho$ thus restricted, we select $\gamma$ such that

$$
0<\gamma<\min \left(\gamma_{0}, \delta^{1 / 2}(12 \pi s)^{-1 / 2}\right),
$$

and consider Lemma 3. The number $\mathscr{S}$ which appears in this lemma is now determined and enables us to choose $N_{0}$ large enough to imply

$$
\text { meas } H_{n} \leqq \mathscr{S} \pi e^{-2 n}<\frac{\delta}{12} \quad\left(n \geqq N_{0}\right) \text {. }
$$

Clearly

$$
H_{n+1} \subset H_{n},
$$

(11.6) $\quad \operatorname{meas}\left(D(\rho, \gamma)-H_{n}\right) \geqq \operatorname{meas}\left(D(\rho, \gamma)-H_{N_{0}}\right)>\pi \rho^{2}-\frac{\delta}{6}$.

Lemma 4 with $\epsilon=k^{(-1 / 2)-(\xi / 2)}$ and $\rho$ as above, yields

$$
\left|\frac{Q_{n}(\zeta)}{Q_{n}(z)}\right| \leqq(|\zeta|+2 \rho)^{n} k^{(1+\xi) n / 2} \quad(n=n(k)),
$$

for

$$
|z| \leqq \rho, \quad z \notin G_{n},
$$

with

$$
\text { meas } G_{n} \leqq 4 \pi e^{2} k^{-1-\xi} \text {. }
$$

From (11.7) and (11.4) we deduce

(11.10) $\left|\frac{Q_{n}(\zeta)}{Q_{n}(z) \zeta^{n}}\right| \leqq\left(1+\frac{2 \rho}{|\zeta|}\right)^{n} \exp \left(\frac{n \log n}{\Sigma+2 \xi}\right) \quad\left(n=n(k), k \geqq K_{0}\right)$. 
Increasing if necessary the value of $K_{0}$, we assume

$$
4 \pi e^{2} \sum_{k=K_{0}}^{\infty} k^{-1-\xi}<\frac{\delta}{6}
$$

so that

$$
\Gamma=\bigcup_{k=K_{0}}^{\infty} G_{n} \quad(n=n(k))
$$

implies

$$
\text { meas } \Gamma<\frac{\delta}{6}
$$

From this point on, all our inequalities will be subject to the conditions

$$
z \in D(\rho, \gamma), \quad z \notin H_{N_{0}}, \quad z \notin \Gamma .
$$

By (11.6) and (11.11) this means that we are excluding from the disk $|z|<\rho$, a measurable set $\Omega_{1}=\Omega_{1}(\rho, \delta)$ with

$$
\text { meas } \Omega_{1}<\frac{\delta}{3} \text {. }
$$

Lemma 3 implies

$$
\frac{1}{\left|V_{n}(z)\right|} \leqq K^{n} \quad\left(z \in\left\{D(\rho, \gamma)-H_{N_{0}}\right\}\right)
$$

where $K>1$ is a bound which depends on several parameters but not on $n$.

The relations (9.1) and (9.2) are valid for $k \geqq K_{0}$ and there only remains to estimate the integrals $J_{n \nu}$, using Lemma 2, (11.10), (11.14) and the inequality

$$
|\zeta-z| \geqq \frac{\gamma}{2} \quad\left(z \in D(\rho, \gamma), \quad \zeta \in \mathscr{C}_{n}, \quad k \geqq K_{0}\right)
$$

We find

$$
\text { (11.15) }\left|\frac{z^{m+n+1}}{V_{n}(z) Q_{n}(z)} J_{0 n}\right| \leqq \frac{2 \rho^{m+n+1} K^{n}}{\gamma|\zeta|^{m-n}|\zeta|^{n_{0}}}\left(1+\frac{2 \rho}{|\zeta|}\right)^{n} \exp \left(\frac{n \log n}{\Sigma+2 \xi}\right)
$$


with

$$
m \geqq n, \quad|\zeta|=\frac{n_{0}^{1 / \sigma_{0}}}{2}, \quad n_{0} \sim \frac{\sigma_{0} n}{\Sigma} \quad(n=n(k), k \rightarrow \infty)
$$

and hence, we deduce from (11.15),

$$
\begin{gathered}
\text { (11.16) }\left|\frac{z^{m+n+1}}{V_{n}(z) Q_{n}(z)} J_{0 n}\right| \leqq K^{n} \exp \left(n \log n\left\{\frac{1}{\Sigma+2 \xi}-\frac{1}{\Sigma}+o(1)\right\}\right) \\
\leqq K^{n} \exp \left(\frac{-\xi n \log n}{\Sigma(\Sigma+2 \xi)}\right) \quad\left(n=n(k), k \geqq K_{0}\right) .
\end{gathered}
$$

Similarly, using (6.5) instead of (6.6), and taking (12) into account, we find that (11.16) holds with $J_{0 n}$ replaced by $J_{\nu n}(\nu=1,2, \cdots, s)$. Hence (9.1) yields the final estimate

$(11.17)\left|f(z)-R_{m n}(z)\right|=\left|\Delta_{k}(z)\right| \leqq(s+1) K^{n} \exp \left(\frac{-\xi n \log n}{\Sigma(\Sigma+2 \xi)}\right)=\eta_{k}$

for

$$
|z| \leqq \rho, \quad z \notin \Omega_{1}, \quad n=n(k) \leqq m(k), \quad k \geqq K_{0} .
$$

Clearly

$$
\eta_{k} \rightarrow 0 \quad(k \rightarrow \infty),
$$

and hence we have proved a "restricted Theorem 1" which requires the additional condition (11.1).

To obtain the general form of the result we consider all the remaining approximants, characterized by

$$
m=m(k)<n(k)=n
$$

and with $m$ and $n$ thus defined, we associate suitable approximants of $1 / f$.

We observe that:

(i) $1 / f$ is regular at the origin and $1 / f(0) \neq 0$;

(ii) the function $1 / f$ has $s+1$ essential singularities at $\alpha_{0}=$ $\infty, \alpha_{1}, \alpha_{2}, \alpha_{s}$, with respective orders $\lambda_{0}, \lambda_{1}, \cdots, \lambda_{s}$;

(iii) the integers $m$ and $n$ appear symmetrically in the conditions (10) and (12).

Consider the Padé approximant 


$$
R_{n m}^{*}(z)=\frac{Q_{n}^{*}(z)}{P_{m}^{*}(z)}
$$

of $1 / f$, where $Q_{n}^{*}(z)$ is of degree $\leqq n, P_{m}^{*}(z)$ is of degree $\leqq m$ and $P_{m}^{*}(z) \not \equiv 0$.

In view of (11.20), the "restricted Theorem 1" is applicable and yields

$$
\left|\frac{1}{f(z)}-\frac{Q_{n}^{*}(z)}{P_{m}^{*}(z)}\right|=\left|\Delta_{k}^{*}(z)\right| \leqq \eta_{k}^{*},
$$

provided

$$
|z| \leqq \rho, \quad z \notin \Omega_{1}^{*}, \quad m=m(k)<n(k)=n, \quad k>K_{0} .
$$

Again, we have

$$
\text { meas } \Omega_{1}^{*}<\frac{\delta}{3}, \quad \eta_{k}^{*} \rightarrow 0 \quad(k \rightarrow \infty) \text {. }
$$

We now construct an open set $\mathscr{G}$ with

$$
\text { meas } \mathscr{G}<\frac{\delta}{3}
$$

and such that $\mathscr{G}$ contains the $s$ essential singularities $\alpha_{\nu}(\nu=1,2, \cdots, s)$ as well as all the poles of $f$ which lie in $|z| \leqq \rho$.

If

$$
|z| \leqq \rho, \quad z \notin \mathscr{G}
$$

we have, for some suitable bound $\omega_{0}$

$$
|f(z)| \leqq \omega_{0}
$$

Hence (11.21), (11.22), and (11.25) imply

$$
\left|\frac{Q_{n}^{*}(z)}{P_{m}^{*}(z)}\right| \geqq \frac{1}{2 \omega_{0}}, \quad Q_{n}^{*}(z) \not \equiv 0 .
$$

We now set

$$
\Omega_{2}=\Omega_{1}^{*} \cup \mathscr{G}
$$


so that

$$
\text { meas } \Omega_{2}<\frac{2 \delta}{3}
$$

By (11.21), (11.26) and (11.27), we see that

$$
|z| \leqq \rho, \quad z \notin \Omega_{2}, \quad m=m(k)<n(k), \quad k \geqq K_{0},
$$

now yield

$$
\left|f(z)-\frac{P_{m}^{*}(z)}{Q_{n}^{*}(z)}\right| \leqq 2 \omega_{0}^{2} \eta_{k}^{*}
$$

By the uniqueness of the Padé table

$$
R_{m n}(z)=\frac{P_{m}(z)}{Q_{n}(z)}=\frac{P_{m}^{*}(z)}{Q_{n}^{*}(z)}
$$

An inspection of (11.17), (11.18), (11.29) and (11.30) shows that we have proved the general form of Theorem 1 . The exceptional set $\Omega$ is

$$
\Omega=\left\{\Omega_{1} \cup \Omega_{2}\right\}
$$

with meas $\Omega<\delta$ (by (11.13) and (11.28)).

\section{REFERENCES}

1. R. J. Arms and A. Edrei, The Padé tables and continued fractions generated by totally positive sequences, Mathematical Essays dedicated to A. J. Macintyre, Ohio University Press, 1970, 1-21.

2. J. S. R. Chisholm, Approximation by sequences of Padé approximants in regions of meromorphy, J. Mathematical Phys., 7 (1966), 39-44.

3. A. Edrei, Sur les déterminants récurrents et les singularités d'une fonction donnée par son développement de Taylor, Compositio Math. 7 (1939), 20-88.

4. - The Padé table of meromorphic functions of small order with negative zeros and positive poles, Rocky Mountain J. Math., 4 (1974), 175-180.

5. E. Hille, Analytic Function Theory, vol. II, Ginn and Company, New York, 1962.

6. O. Perron, Die Lehre von den Kettenbrüchen, 3rd. ed., vol. 2, B. G. Teubner, Stuttgart, 1957.

7. G. Pólya, Über gewisse notwendige Determinantenkriterien für die Fortsetzbarkeit einer Potenzreihe, Math. Annalen, 99 (1928), 687-706.

8. C. Pommerenke, Padé approximants and convergence in capacity, J. Math. Analysis and Appl., 41 (1973), 775-780.

9. E. C. Titchmarsh, The theory of Functions, 2nd. ed., Oxford University Press, Oxford, 1952.

10. G. Valiron, Fonctions entières d'ordre fini et fonctions méromorphes, L'Enseignement Mathématique, Genève, 1960.

Received November 6, 1973. The research of the author was supported by a grant from the National Science Foundation GP-33175

SYRACUSE UNIVERSITY 
